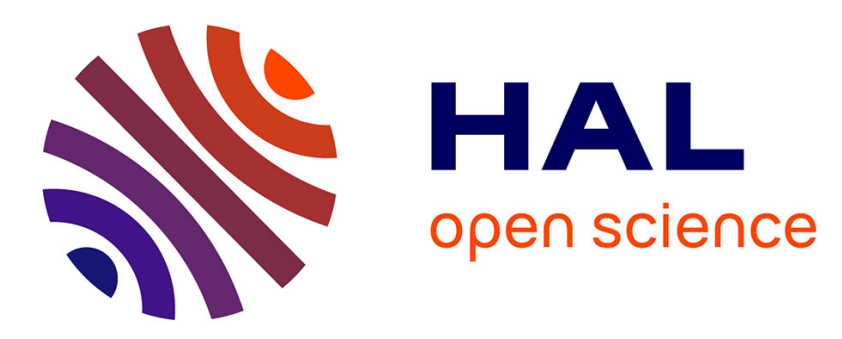

\title{
Des machines, des vaches et des hommes. Projets culturels, acteurs et territoires dans un espace rural en crise. L'exemple de la Thiérache
}

Emmanuelle Bonerandi, Camille Hochedez

\section{- To cite this version:}

Emmanuelle Bonerandi, Camille Hochedez. Des machines, des vaches et des hommes. Projets culturels, acteurs et territoires dans un espace rural en crise. L'exemple de la Thiérache. Norois, 2007, Patrimoine, culture et construction identitaire dans les territoires ruraux, 204 (3), pp.25-37. 10.4000/norois.1413. halshs-01343721

\section{HAL Id: halshs-01343721}

https://shs.hal.science/halshs-01343721

Submitted on 17 Jul 2016

HAL is a multi-disciplinary open access archive for the deposit and dissemination of scientific research documents, whether they are published or not. The documents may come from teaching and research institutions in France or abroad, or from public or private research centers.
L'archive ouverte pluridisciplinaire HAL, est destinée au dépôt et à la diffusion de documents scientifiques de niveau recherche, publiés ou non, émanant des établissements d'enseignement et de recherche français ou étrangers, des laboratoires publics ou privés. 
Emmanuelle Bonerandi et Camille Hochedez

\title{
Des machines, des vaches et des hommes. Projets culturels, acteurs et territoires dans un espace rural en crise : l'exemple de la Thiérache
}

\begin{abstract}
Avertissement
Le contenu de ce site relève de la législation française sur la propriété intellectuelle et est la propriété exclusive de l'éditeur.

Les œuvres figurant sur ce site peuvent être consultées et reproduites sur un support papier ou numérique sous réserve qu'elles soient strictement réservées à un usage soit personnel, soit scientifique ou pédagogique excluant toute exploitation commerciale. La reproduction devra obligatoirement mentionner l'éditeur, le nom de la revue, l'auteur et la référence du document.

Toute autre reproduction est interdite sauf accord préalable de l'éditeur, en dehors des cas prévus par la législation en vigueur en France.
\end{abstract}

\section{revues.org}

Revues.org est un portail de revues en sciences humaines et sociales développé par le Cléo, Centre pour l'édition électronique ouverte (CNRS, EHESS, UP, UAPV).

\author{
Référence électronique \\ norois. 1413 \\ Éditeur : Presses universitaires de Rennes \\ http://norois.revues.org \\ http://www.revues.org \\ Document accessible en ligne sur : http://norois.revues.org/1413 \\ Ce document est le fac-similé de l'édition papier. \\ ( ) Tous droits réservés
}

Emmanuelle Bonerandi et Camille Hochedez, « Des machines, des vaches et des hommes. Projets culturels, acteurs et territoires dans un espace rural en crise : l'exemple de la Thiérache », Norois [En ligne], 204 | 2007/3, mis en ligne le 01 septembre 2009, consulté le 03 avril 2014. URL : http://norois.revues.org/1413 ; DOI : 10.4000/ 


\title{
DES MACHINES, DES VACHES ET DES HOMMES \\ Projets culturels, aCteurs et territoires
}

\section{DANS UN ESPACE RURAL EN CRISE : L'EXEMPLE DE LA THIÉRACHE}

\author{
Emmanuelle Bonerandi, Camille Hochedez \\ GÉophile - UMR CNRS Environnement, VilLes, SociétéS \\ (Université de Lyon - École Normale Supérieure Lettres et Sciences humaines), \\ 15 parvis René-Descartes, BP 7000 - 69342 LyON cedex 07 \\ Emmanuelle.Bonerandi@ens-lsh.fr, Camille.Hochedez@ens-lsh.fr
}

\begin{abstract}
RÉSUMÉ
La dimension territoriale des faits culturels constitue une entrée importante pour analyser les dynamiques et recompositions des espaces ruraux et de leurs sociétés. L'article examine la démarche 'projet culturel' dans les espaces ruraux anciennement industrialisés. Dans ces espaces, considérés comme des "déserts culturels", les projets culturels constituent l'un des moyens essentiels pour des acteurs de travailler ensemble, pour des populations de se rencontrer et de partager. La construction d'un lien social et territorial renowvelé et réactivé par le projet culturel constitue la problématique de l'article. Ces interrogations générales sont abordées au travers d'une recherche sur le rôle des projets culturels dans le développement socio-territorial d'une région rurale anciennement industrialisée, la Thiérache. Il s'agit de cerner les facteurs de recomposition socio-territoriale contenus dans les projets culturels (nature du projet, assise territoriale, organisation des acteurs, temporalité, participation de la population, effet mobilisateur) et d'en évaluer la pertinence au regard de la construction du lien social.
\end{abstract}

MotS CLÉS : Développement territorial - Espace rural - France - Géographie sociale et culturelle-Projet culturel - Thiérache.

\section{ABSTRACT}

Machines, cows and men: cultural projects, actors and territories in an, ill favoured country space: the example of the Thiérache

Territorial dimension of cultural facts is an important point of view to analyze spatial and social rural dynamics. This paper addresses cultural projects in old industrialized rural areas as a way for local forces to work together, for population to meet and to share. Cultural projects are considerated in their capacities to build a new social and territorial link. A focus is given through the example of Thierache, small rural area in North-East of France. Cultural projects are examined according to their nature, territorial base, local forces organization, temporality, involvement of population.

KEY WORDS : Cultural Project-France - Rural Areas - Social and Cultural Geography - Territorial Development - Thiérache. 
La définition et la mise en œuvre de projets culturels en milieu rural permettent de tester la capacité d'expression collective des habitants. Généralement, les études portent sur les projets menés dans le cadre d'espaces ruraux à fortes aménités patrimoniales, à travers la confrontationcollaboration entre autochtones et néo-ruraux. Cette focalisation laisse souvent dans l'ombre les espaces ruraux anciennement industrialisés; la valorisation du patrimoine industriel s'opérant davantage dans les espaces à dominante urbaine. Ces espaces disqualifiés sont souvent considérés comme des « déserts culturels». Cependant, des projets culturels les animent, qui constituent souvent l'un des moyens essentiels pour des acteurs de travailler ensemble, pour des populations de se retrouver. La problématique de la construction d'un lien social renouvelé par le projet culturel n'en est posée qu'avec plus d'acuité. Elle invite à dépasser l'alternative « culture du loto » versus « culture élitiste » pour envisager la culture comme un bien partagé par tous, un vecteur du vivre ensemble.

L'objectif est ici d'interroger la capacité du projet culturel à construire du territoire et à recréer du lien social à travers l'analyse de la situation observée en Thiérache. L'étude de quatre projets permet de cerner les facteurs de recomposition socio-territoriale et d'en évaluer la pertinence au regard de la construction du lien social. L'analyse permet d'évaluer leur rôle de levier de développement territorial et social.

La Thiérache est engagée depuis plus d'une génération dans une crise polymorphe et cumulative qui touche à la fois le territoire, les activités et les populations, au point de se demander s'il s'agit encore d'une crise ou d'une mutation (Bonerandi, 1999). Le territoire présente le double handicap d'une situation de marge géographique et de confins territorial : l'inscription dans le quart nord-est de la France définit un angle mort du territoire national, éloigné des principaux centres urbains régionaux. Le territoire est fragmenté entre trois départements, trois régions et deux pays (fig. 1).

L'hémorragie démographique est ancienne : de 1962 à 1999, selon un rythme quasi-régulier, la Thiérache est passé de 193455 à 161235 habitants (-16,5\%). Le taux de chômage atteint 13,4\% de la population active en 2003, soit 4 points au-dessus de la moyenne nationale. Il dépasse $20 \%$ dans les communes industrielles et urbaines d'Hirson et de Fourmies. Il résulte de la disparition de l'ancien système productif, d'origine exogène, fondé sur la métallurgie et le textile. Le niveau de qualification des populations est particulièrement faible. Les indicateurs de pauvreté se recoupent et s'additionnent (Bonerandi, 2007).

Le développement économique des cités à vocation industrielle et ouvrière a induit l'émergence d'une société bipolaire : d'une part, une catégorie relativement préservée, constituée des populations aujourd'hui retraitées et des populations ayant un emploi, notamment dans le secteur public, et d'autre part, les populations en difficulté, présentes notamment parmi les populations en âge d'être actives, qui n'ont pas ou peu connu le monde du travail et qui se maintiennent grâce à l'aide sociale. Plus généralement, les relations complexes et concurrentes entretenues entre une sphère agricole indépendante et une sphère industrielle ouvrière entérinent une partition socioéconomique et culturelle du territoire.

\section{Le projet culturel pour penser \\ les recompositions socio-territoriales en milieu rural}

La dimension culturelle participe de l'être rural de ce tournant de siècle (Jean, 2001). On semble assister à un « renouveau » culturel, hybridation entre la demande d'une offre culturelle diversifiée de la part des habitants, permanents ou temporaires, la volonté des acteurs locaux d'assurer l'attractivité de leur territoire et la demande sociale de valorisation du patrimoine rural.

La création artistique a conquis la campagne. Les festivals, bals, fêtes populaires et autres animations à dimension patrimoniale entretiennent la dimension culturelle et festive des espaces ruraux (Crozat, 1998; Di Méo, 2001). Même si la définition d'une politique culturelle dédiée aux espaces ruraux a disparu de l'agenda des services centraux de l'État, nombreuses ont été les actions 


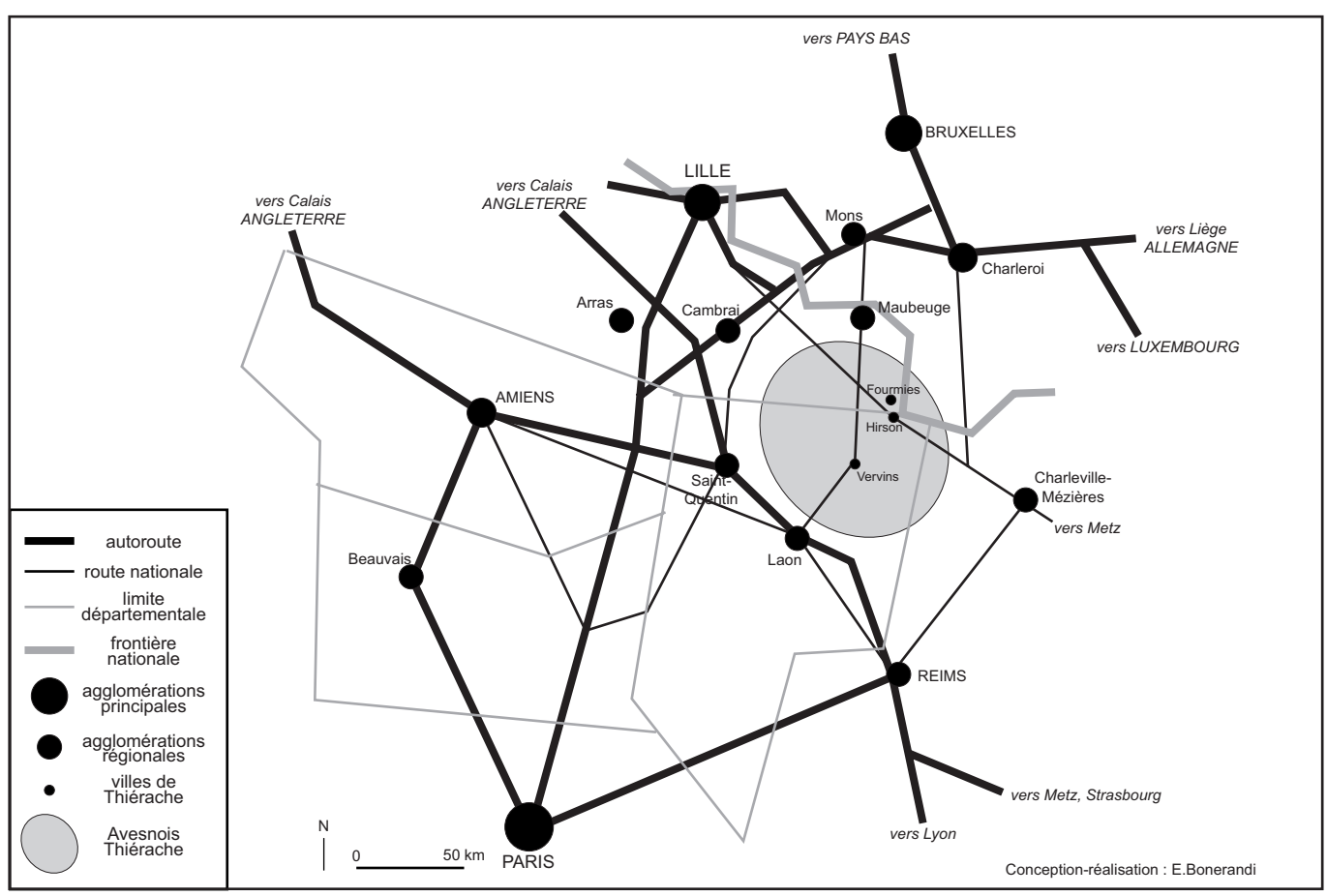

Figure 1 : La Thiérache, un angle mort du territoire français Localization of Thierache

initiées par le ministère, tels la création du Fonds d'Initiative Culturelle dans les années 1970 ou la plus récente création des Pôles d’Économie du Patrimoine (Virassamy, 2002). La décentralisation a permis aux collectivités territoriales d'assurer et de soutenir la promotion culturelle des territoires ruraux (Moulinier, 2003). Enfin, il convient de rappeler le rôle joué par les associations locales et par les mouvements d'envergure nationale, tels les écomusées, les musées d'Arts et Traditions Populaires ou la Confédération Nationale des Foyers Ruraux. Cette dernière est emblématique de la dimension sociale de la culture qui agit comme un liant, « ce par quoi tient l'ensemble » (Bonnemaison, 2000). Le mouvement des Foyers Ruraux a largement œuvré pour faire du développement culturel le moteur du développement économique et social. Conçue durant la Résistance et officialisée en 1946, la Confédération Nationale des Foyers Ruraux entend ouvrir les campagnes à toutes les dimensions du progrès et de la culture. Associations d'éducation populaire, d'éducation permanente et citoyenne, ils contribuent à l'animation et au développement culturels, sociaux et économiques du milieu rural. Les buts en sont la participation et l'apprentissage, inséparables de la création afin de «permettre l'accès à la culture du plus grand nombre ».

La dimension territoriale des faits culturels permet d'analyser les dynamiques et recompositions des espaces ruraux et de leurs sociétés. La notion de territoire fait appel à la culture. Nous entendons ici la culture dans une acception large, celle de la façon dont les hommes se comportent, agissent, communiquent en société. Que ce soit dans le domaine de la perception du territoire, de ses représentations, de son appropriation symbolique, la construction territoriale résulte de la culture de la population qui y vit. Ce lien est à double sens : d'un côté le territoire produit du culturel (ainsi la « mise en mythe du paysage » résulte d'une opération culturelle qui s'élabore à partir des caractéristiques géographiques); de l'autre, le culturel produit du territoire par l'usage d'emblèmes, de symboles. L'ambition est ici de s'intéresser plus spécifiquement au projet culturel, comme signe de la « place de 
la culture dans l'aménagement du territoire comme facteur d'attractivité, de dynamisme économique et surtout de cohésion sociale» (ministère de la Culture et de la Communication, 1998).

Le terme de projet a connu un succès retentissant. Les récentes lois d'aménagement du territoire intiment l'ordre aux territoires d'élaborer des projets, qui seraient bien inspirés de s'ériger en projet de territoire. Le projet relève de ce qu'on a l'intention de faire, insistant ainsi sur l'intention des acteurs porteurs du projet et sur la temporalité de l'action, en tant qu'action de projection dans l'avenir. Partant du présupposé que tout projet est un projet de société, élaboré par une société pour une société, la réflexion porte sur son inscription dans un contexte socio-spatial et sur ses effets sociaux et territoriaux.

Le rôle de la culture dans le développement socio-territorial interroge la notion de projet culturel à plusieurs titres :

- le projet culturel constitue une étape, par essence temporaire, voué à disparaître pour laisser place à sa concrétisation ;

- le projet culturel correspond à un moment particulier, celui où se regroupent des acteurs d'origines diverses (géographique, socio-professionnelle, générationnelle...) dans le but d'élaborer une œuvre commune, de construire un fait de consensus;

- par-delà la déclinaison en actions spécifiques, le projet culturel vaut par sa dimension performative dans une double acceptation de «faire parler sur » et de «faire parler entre »;

- le projet culturel représente une opportunité pour les acteurs de construire un projet à la fois territorial et socio-spatial;

- le projet culturel peut constituer un moyen de conscientisation d'un groupe social d'appartenir à un même territoire, de partager une même identité;

- le projet culturel représente, enfin, la projection d'une société dans l'avenir.

Enfin, même si le propos de J. Rigaud (1996) ne s'applique pas spécifiquement aux espaces ruraux, le culturel occupe une place centrale dans le projet de société et dans les choix politiques des acteurs, car "qu'il s'agisse de maintenir ou de consolider le lien social, d'assurer une égalité effective entre les êtres, de garantir les conditions de leur dignité et de leur épanouissement, aucun de ces choix qui impliquent une volonté politique ne doit ignorer la culture, mais bien en faire un facteur-clé des décisions ».

\section{La culture, de la confiture aux cochons?}

Les politiques de développement initiées par les collectivités locales en Thiérache accordent peu de place au développement culturel. Ainsi, dans le Contrat de Plan État-Région de Picardie (2000-2006), la culture n'est présente que sous le volet «mise en valeur du cadre de vie », dans une optique de valorisation touristique du territoire. Pourtant, la multiplicité des initiatives locales et des manifestations culturelles prouve que la Thiérache n'est pas un désert culturel. Qu'il s'agisse de la valorisation des produits de terroir (AOC Maroilles, cidre fermier, et autres foire aux fromages ou fête du lait) ou de la préservation du patrimoine bocager (Parc Naturel Régional de l'Avesnois), la Thiérache a fait des événements culturels un moyen de valoriser son territoire (Delfosse, 2003; Amas, 1997; Dubois, 1995). Mais la culture (ou son étude) semble se réduire au patrimoine rural, et surtout agricole. Choisir des projets ayant des thèmes aussi variés que l'industrie textile, l'utopie socialiste ou les vaches, interroge ce qui fonde la réussite du projet culturel dans un milieu rural de ce type.

Quatre projets ont été retenus, en fonction de leur assise territoriale, de leur durabilité et de la participation de la population : le Festivache, l'Écomusée de la Région de Fourmies-Trélon, Utopia (familistère Godin) et la Saison Culturelle des Transfrontalières. Ces projets émanent de territoires différents (tableau 1), concernant respectivement une communauté de communes, un réseau, un 
site et une Eurorégion en constitution. Les thèmes sont variés : rural et agricole (le Festivache), mais aussi industriel (l'Écomusée), concernant un patrimoine de renommée internationale (Utopia et le familistère) ou lié à un financement spécifique (programme Interreg pour les Transfrontalières).

\begin{tabular}{|c|c|c|c|c|}
\hline Projet culturel & Écomusée & Festivache & Utopia & Transfrontalières \\
\hline $\begin{array}{l}\text { Date } \\
\text { de création }\end{array}$ & $\begin{array}{l}\text { - } 1980 \text { (exposition de } \\
\text { préfiguration) } \\
\text { • ouverture progressive } \\
\text { jusqu'en } 1986\end{array}$ & • 2001 & $\begin{array}{l}\text { - Projet non réalisé } \\
\text { l'étude de référence } \\
\text { date de } 1996\end{array}$ & • 1995 \\
\hline $\begin{array}{l}\text { Porteurs du } \\
\text { projet }\end{array}$ & $\begin{array}{l}\text { - directeur } \\
\text { - Syndicat Mixte pour } \\
\text { le Développement de la } \\
\text { Thiérache }\end{array}$ & $\begin{array}{l}\text { - directeur de Tac Tic } \\
\text { Animation } \\
\text { - maire de Sorbais et } \\
\text { vice-président de la } \\
\text { Communauté de Com- } \\
\text { mune de Thiérache du } \\
\text { Centre (CCTC) } \\
\text { - CCTC }\end{array}$ & $\begin{array}{l}\text { - député-maire } \\
\text { de Vervins } \\
\text { - conservateur } \\
\text { du musée }\end{array}$ & $\begin{array}{l}\text { - maire d'Hirson } \\
\text { - directrice du centre } \\
\text { culturel Sudhaina } \\
\text { (entité de Chimay) }\end{array}$ \\
\hline $\begin{array}{l}\text { Acteurs } \\
\text { du quotidien }\end{array}$ & $\begin{array}{l}\text { - } 40 \text { salariés et } 150 \\
\text { bénévoles répartis sur } 6 \\
\text { antennes }\end{array}$ & $\begin{array}{l}\text { - Tac Tic Animation } \\
\text { - Artistes } \\
\text { - Population }\end{array}$ & $\begin{array}{l}\text { - Syndicat Mixte du } \\
\text { Familistère Godin } \\
\text { (Département de } \\
\text { l'Aisne et Ville de } \\
\text { Guise) }\end{array}$ & $\begin{array}{l}\text { - Service culturel de la } \\
\text { ville d'Hirson } \\
\text { - Services techniques } \\
\text { des trois villes partici- } \\
\text { pantes (Chimay, Hir- } \\
\text { son, Fourmies) }\end{array}$ \\
\hline $\begin{array}{l}\text { Principaux } \\
\text { financeurs }\end{array}$ & $\begin{array}{l}\text { - communes adhé- } \\
\text { rentes } \\
\text { - Département du } \\
\text { Nord } \\
\text { - Région Nord-Pas-de- } \\
\text { Calais } \\
\text { - État } \\
\text { - Europe }\end{array}$ & $\begin{array}{l}\text { - CCTC } \\
\text { - Département de } \\
\text { l'Aisne } \\
\text { - Région Picardie } \\
\text { - CAF } \\
\text { - «Traditions en fête » }\end{array}$ & $\begin{array}{l}\text { - Ville de Guise } \\
\text { - Communauté de } \\
\text { Communes «Région } \\
\text { de Guise-Thiérache } \\
\text { d'Aumale » } \\
\text { - Département de } \\
\text { l'Aisne } \\
\text { - Région Picardie } \\
\text { - État } \\
\text { - Europe }\end{array}$ & $\begin{array}{l}\text { - Union Européenne } \\
\text { (INTERREG) à 50\% } \\
\text { • communes d'Hirson, } \\
\text { Chimay et Fourmies }\end{array}$ \\
\hline Budget & & 53650 euros & 13 millions d'euros & 745310 euros \\
\hline $\begin{array}{l}\text { Fréquentation / } \\
\text { participation }\end{array}$ & $\begin{array}{l}\text { • } 44499 \text { visiteurs en } \\
2002\end{array}$ & $\begin{array}{l}\text { - } 1800 \text { vaches } \\
\text { - } 20 \text { communes parti- } \\
\text { cipantes } \\
\text { - } 5000 \text { visiteurs toutes } \\
\text { manifestations confon- } \\
\text { dues }\end{array}$ & $\begin{array}{l}\text { - Actuelle : } 20000 \\
\text { visiteurs/an } \\
\text { - Projetée : } 100000 \\
\text { visiteurs/an }\end{array}$ & $\begin{array}{l}-72011 \text { spectateurs } \\
\text { toutes manifestations } \\
\text { confondues depuis } \\
1996\end{array}$ \\
\hline Temporalité(s) & $\begin{array}{l}\text { - Expositions perma- } \\
\text { nentes et temporaires } \\
\text { - Manifestations ponc- } \\
\text { tuelles autour du patri- } \\
\text { moine (randonnées...) }\end{array}$ & $\begin{array}{l}\text { - Festival annuel allant } \\
\text { de la mi-juin à la mi- } \\
\text { juillet } \\
\text { - Organisation de } \\
\text { temps forts (week-end } \\
\text { et expositions) } \\
\text { - Vaches exposées } \\
\text { toute l'année }\end{array}$ & $\begin{array}{l}\text { - Musée permanent } \\
\text { - Événementiel depuis } \\
2000 \text { : les Fêtes du } \\
\text { ler mai }^{\text {er }}\end{array}$ & $\begin{array}{l}\text {-Spectacles pendant } \\
\text { l'année scolaire }\end{array}$ \\
\hline
\end{tabular}

Tableau 1 : Présentation des projets culturels (Source : Hochedez C., 2004, enquêtes) The cultural projects: a presentation 
- Le Festivache, festival d'art bovin contemporain, se déroule entre la mi-juin et la mi-juillet. La population est invitée à réaliser des vaches de toute forme, exposées devant les maisons. À cette exposition en plein air sont associés des « temps forts » (concerts, fêtes, randonnées...). Porté par le centre culturel de la communauté de communes de Thiérache du Centre (CCTC), l'objectif est de donner une légitimité identitaire à la CCTC, qui est souvent une structure méconnue des habitants. La première édition a eu lieu en 2001.

- L'Écomusée est un projet devenu réalité culturelle (photo 1). Cet équipement fonctionne comme un réseau de six antennes et de deux musées, progressivement constitué entre 1982 et 1986. Chacun traite d'un thème représentatif de l'identité thiérachienne (maison du bocage, musée du verre). L'antenne principale de Fourmies, est axée sur le patrimoine industriel textile et la vie sociale qui y est associée. Outre les expositions permanentes, les différents sites proposent des animations pédagogiques à destination des scolaires et des manifestations autour du patrimoine, de type randonnée de découverte de la faune et de la flore.

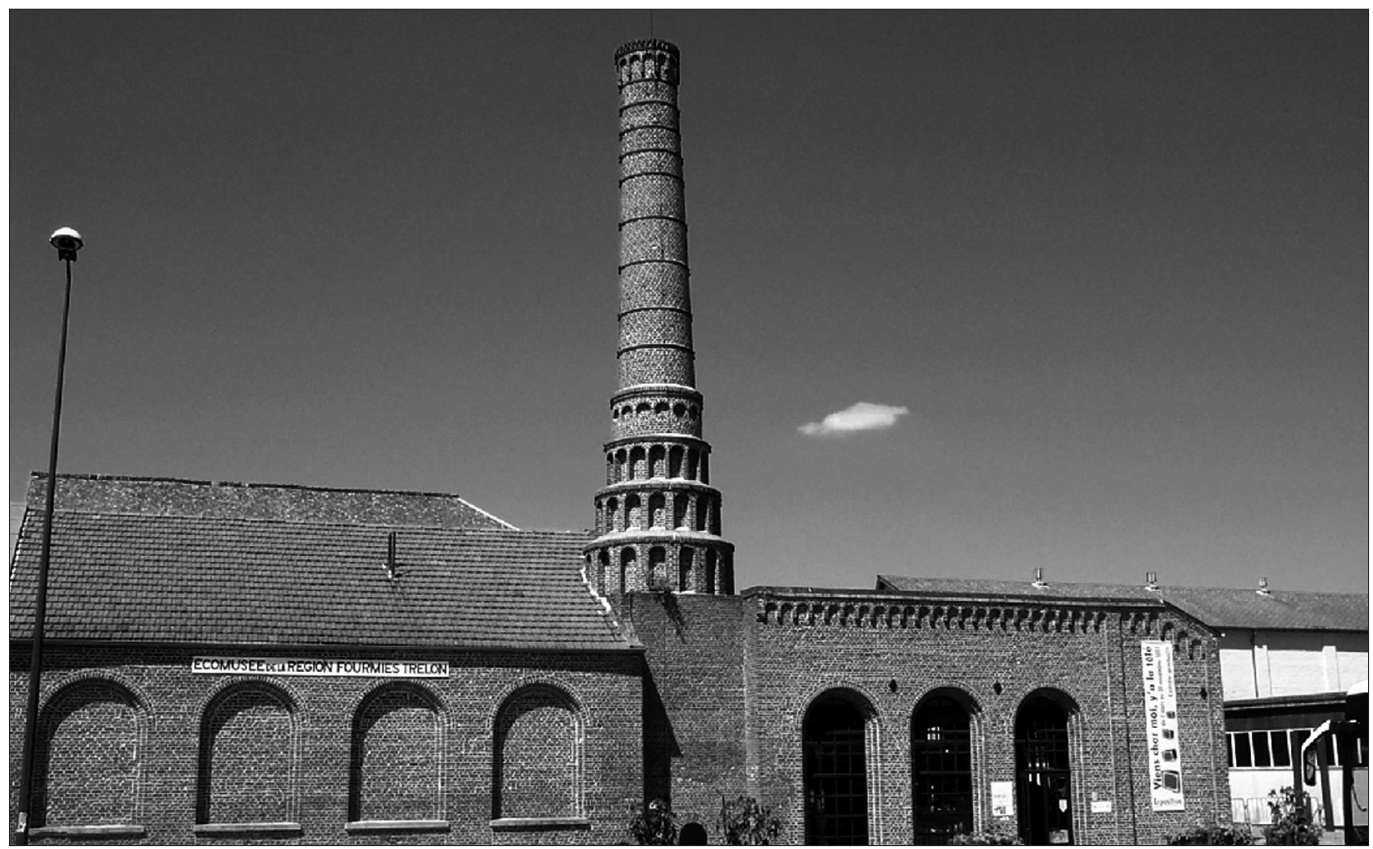

Photo 1 : L'Écomusée du Textile et de la Vie sociale à Fourmies

L'écomusée prend place dans l'ancienne filature de laine peignée Prouvost-Masurel rachetée par la municipalité, puis louée à l'écomusée pour un euro symbolique. Les travaux de réhabilitation ont permis d'installer une exposition permanente sur la production textile et la vie sociale du XIX ${ }^{\mathrm{e}}$ siècle, un centre de documentation, une boutique, un café, un espace d'expositions temporaires, ainsi que des bureaux réservés à l'administration (cliché C. Hochedez - juillet 2003).

Eco Museum (or heritage center) for textile and social life in Fourmies

This heritage center is located within the former wool factory which has been bought by the city for one symbolic Euro. Renovation works have allowed the setting of an exhibition dealing with textile production and social life during the $19^{\text {th }}$ century. An information desk and documentation center, a shop, a café and some offices (for management) have also been included in the building.

- Le projet Utopia a pour objectif la valorisation scientifique, culturelle et touristique du familistère Godin de Guise. Il reprend l’identité industrielle et ouvrière du site, entend réhabiliter la totalité du complexe pour en faire un site touristique majeur à l'échelle de l'Europe du Nord.

- Les Transfrontalières n'ont pas de thème spécifique, elles sont une programmation culturelle commune aux trois villes d'Hirson (Aisne), de Chimay (Belgique) et plus récemment de Fourmies 
(Nord). Les spectacles sont variés, aussi bien des concerts, des pièces de théâtre, des spectacles de danse. La programmation propose également des expositions et des festivités comme les « Transfrontalières » (festival de musique organisé à Hirson à la Pentecôte). La manifestation se veut le premier jalon de la constitution d'une Eurorégion.

Ces quatre projets sont représentatifs de la volonté des acteurs locaux de donner une dimension culturelle aux projets de développement. Ils représentent deux types de projet culturel: l'événementiel et l'équipement pérenne. Par-delà les différences, l'objectif est de donner une autre identité et une autre image à la Thiérache.

\section{Des projets pour des territoires?}

Malgré leur volonté affichée de construire une cohésion territoriale d'ensemble, aucun projet ne semble faire émerger de territoire commun. Chaque projet construit sa propre territorialité, selon des formes diverses. Une comparaison du tableau 2 et de la figure 2 montre le décalage entre les territoires projetés et l'assise territoriale réelle.

\begin{tabular}{|c|c|c|c|c|}
\hline Projet culturel & Écomusée & Festivache & Utopia & Transfrontalières \\
\hline Territoire (s) & $\begin{array}{l}\bullet 6 \text { antennes }+2 \\
\text { musées associés } \\
\text { • territoire géogra- } \\
\text { phique de l'Écomu- } \\
\text { sée (17 communes) } \\
\text { • champ d'inter- } \\
\text { vention contractuel } \\
\text { projeté (Avesnois) } \\
\text { •réseau national } \\
\text { des écomusées de } \\
\text { France } \\
\bullet \text { Jumelage avec } \\
\text { Coptree (Angle- } \\
\text { terre) }\end{array}$ & $\begin{array}{l}\bullet \text { Dorsale nord-sud } \\
\text { de villages impli- } \\
\text { qués } \\
\bullet \text { Volonté d'élar- } \\
\text { gissement à toute } \\
\text { la Thiérache de } \\
\text { l'Aisne et à la Bel- } \\
\text { gique }\end{array}$ & $\begin{array}{l}\text { - Le Familistère, un } \\
\text { quartier de Guise } \\
\text { - La Thiérache de } \\
\text { l'Aisne } \\
\text {-Pas d'assise terri- } \\
\text { toriale }\end{array}$ & $\begin{array}{l}\text { - Un réseau de trois } \\
\text { villes (Chimay, Hir- } \\
\text { son, Fourmies) } \\
\text { - La Thiérache au } \\
\text { sens large } \\
\text { - Une région } \\
\text { européenne trans- } \\
\text { frontalière (Euro- } \\
\text { région): échelle en } \\
\text { construction }\end{array}$ \\
\hline
\end{tabular}

Tableau 2 : L'assise territoriale des projets (Source : Hochedez C., 2004, enquêtes)

Territorial settings of the projects

- Utopia constitue un projet de site. Son assise territoriale est ponctuelle; elle se résume à un quartier de Guise, véritable enclave socio-spatiale, lieu emblématique. L'identité territoriale du Familistère n'a pas de réel contenu, dans la mesure où la population et la majorité des acteurs locaux ne se sentent pas concernés par le projet; ils estiment que les investissements apportent surtout à la commune de Guise, qui en a d'ailleurs profité pour réaliser des travaux d'embellissement du centre-ville sur l'enveloppe budgétaire du projet Utopia.

- L'Écomusée et les Transfrontalières ont une assise territoriale réticulaire. Le réseau de l'Écomusée est consolidé, du fait de son ancienneté, mais aussi parce que totalement inscrit dans le département du Nord. Il s'appuie sur les 17 communes adhérentes, avec lesquelles l'Écomusée a signé une " convention de territoire », sur la base d'une cotisation communale annuelle au prorata du nombre d'habitants (45 euros par an et par habitant). Sa reconnaissance lui permet de collaborer avec d'autres structures régionales, comme le Parc Naturel Régional de l'Avesnois, la fédération touristique « Tourisme en Avesnois », et les structures porteuses du futur pays SambreAvesnois. Porteur de projet de toutes ces institutions, l'Écomusée a joué un rôle d'effet-levier. Mais 


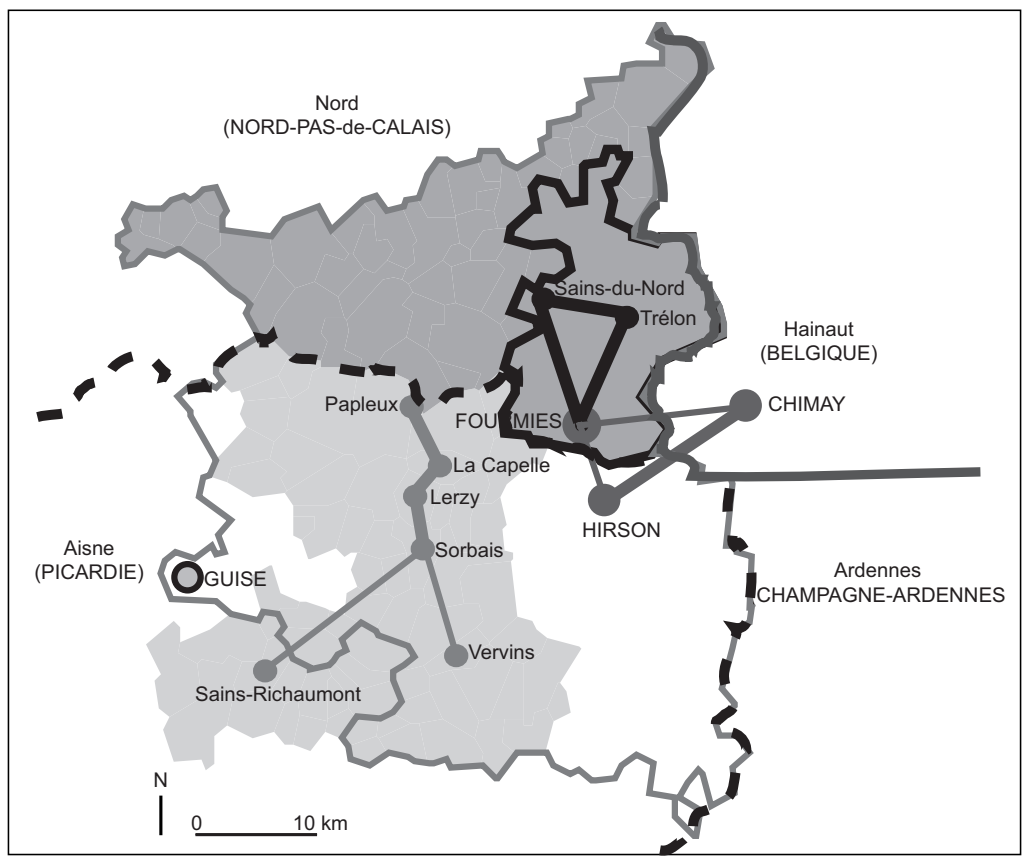

1. Des projets culturels aux logiques territoriales multiples 1.1. Du site ...
8 antennes principales de l'Ecomusée de Forumies-Trélon
8 projet utopia (Familistère de Guise)
pôles des Transfrontalières
noyaux du Festivache

1.2. ... au réseau

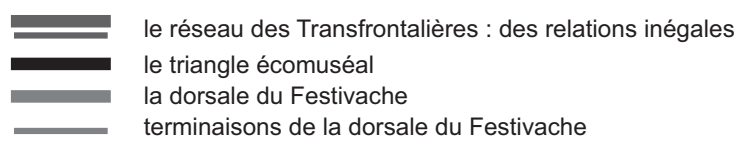

2. Des projets qui transcendent rarement les territoires de projet

frontière franco-belge
limites départementales
territoire de l'Ecomusée
"champ d'intervention géographique" de l'Ecomusée
territoire théorique du Festivache: la C.C.T.C.
limites de I'A.O.C. Maroilles

Conception-réalisation : E.Bonerandi, C.Hochedez

Figure 2 : Projets culturels et territoires en Thiérache Cultural projects and territories in Thiérache

son territoire est déséquilibré; l'antenne-mère de Fourmies capte l'essentiel des flux de visiteurs et des investissements, au point que d'autres antennes sont menacées dans leur existence même. Ce réseau combine différents niveaux, car il est lui-même inséré dans le réseau national des Écomusées, et il collabore avec des musées étrangers (jumelage avec le musée de Coptree en Angleterre). Enfin, tributaire de la politique culturelle régionale, il a plus de lien avec Lille qu’avec la Thiérache de l'Aisne, les tentatives de coopération ayant échoué. Dans les deux cas, la carte fait apparaître 
des réseaux déséquilibrés et fortement polarisés par une petite ville : Fourmies pour l'Écomusée, Hirson pour les Transfrontalières.

- Le cas des Transfrontalières fait apparaître un réseau inachevé et fortement dissymétrique. Les relations sont beaucoup plus fortes entre Hirson et Chimay, véritable axe de structuration de cet espace transfrontalier, qu'avec Fourmies, rattaché tardivement. L'opération n'en est encore qu'au stade de conscientisation des populations. Un système de navettes en car a été mis en place, en espérant qu'il joue un rôle incitatif pour d'autres déplacements. Or, la population ne se déplace occasionnellement que pour des spectacles. Le territoire est lacunaire. Pour combler ces «vides », les organisateurs prévoient d'élargir la zone d'influence des manifestations à d'autres communes, comme celles de Momignies (Belgique), de Signy-le-Petit (Ardennes), d'Anor (Nord) et de tout le canton d'Hirson, afin de donner une réelle consistance à l'Eurorégion, en y intégrant la partie ardennaise.

- Le Festivache constitue l'exemple le plus abouti de réussite territoriale. Son assise territoriale correspond à peu près aux limites de la CCTC. En ce sens, il participe pleinement à la construction identitaire du territoire de projet. À partir de la mi-juin, le territoire de la CCTC se couvre progressivement de vaches d'art de toutes formes et de toutes matières, les plus belles étant récompensées par un prix. En 2003, il y a eu 1800 représentations de vaches dans les communes du territoire de la CCTC, contre 1100 en 2002, soit une augmentation de plus de $60 \%$. La participation aux «temps forts » est importante pour les manifestations mettant en scène la ruralité («Ferme au cœur de la ville», conférence sur les races de vaches, course de moissonneusesbatteuses). Le succès est tel que certaines communes hors de la CCTC ont été associées. C'est le cas des communautés de communes voisines (Région de Guise et de la Thiérache d'Aumale, Portes de la Thiérache, Pays des Trois Rivières) qui participent financièrement à l'organisation de la randonnée cycliste et pédestre "la Thiérachienne », qui se tient durant le Festivache. Lors de l'édition 2003, le départ a été donné de Chimay (Belgique). La carte fait apparaitre une dorsale de villages allant de la Flamengrie au nord à Saint-Algis au sud, où le nombre de vaches réalisées est plus important, et où l'implication des élus est plus forte. Les communes constituant ce noyau dur ont pratiquement toutes moins de 200 habitants, à l'exception de la Flamengrie. Comme le souligne le maire de Papleux : «Pour les petites communes, participer, c'est une façon de prouver qu'il y a encore de la vie. » L'émulation créée par le concours récompensant les plus belles réalisations joue à plein. D'une édition à l'autre, les communes fortement impliquées dans la réalisation de vaches ou dans l'organisation d'un temps fort varient peu. Le Festivache est désormais un événement attendu; il a réussi à faire connaître la CCTC à ses habitants, à faire travailler ensemble les acteurs locaux en leur démontrant l'utilité d'une coopération intercommunale. La création d'un logo a donné une identité, un symbole au territoire.

\section{Projet culturel, projet social : la culture pour vivre ensemble?}

Aucun des projets n'arrive à concerner l'ensemble de l'espace d'étude. La culture n'échappe pas aux effets de discontinuité territoriale. Si chaque projet rencontre des limites qui lui sont propres (tableau 3), ils ont en commun de devoir composer avec le faible nombre des porteurs de projets, inhérent à la faible densité relative de la Thiérache, et surtout au manque de renouvellement et à la faible ouverture de la société locale. Les enquêtes auprès des porteurs de projet ont clairement pointé le manque de structure d'encadrement, qui nuit notamment à une bonne communication, la difficulté à recruter des animateurs, la lassitude et, plus généralement, la difficulté à faire soutenir des projets culturels sur un territoire périphérique. L'absence de renouvellement démographique par arrivée de populations extérieures ne permet pas d'observer l'émergence d'une demande culturelle renouvelée. Les projets culturels sont avant tout des projets d'élus locaux, ils n'émanent pas de la population. 


\begin{tabular}{|c|c|c|c|}
\hline Écomusée & Festivache & Utopia & Transfrontalières \\
\hline $\begin{array}{l}\text { - Pérennité: comment } \\
\text { assurer la continuité du } \\
\text { phénomène } \\
\text { - Difficulté financière: } \\
\text { assurer une part maxi- } \\
\text { male d'autofinancement }\end{array}$ & $\begin{array}{l}\text { - Communication: tra- } \\
\text { vail sur l'image, et pas } \\
\text { sur le contenu } \\
\text { - Lassitude } \\
\text { - Concilier participation } \\
\text { de masse et qualité artis- } \\
\text { tique }\end{array}$ & $\begin{array}{l}\text { - Résistance des habi- } \\
\text { tants du Familistère } \\
\text { - Difficulté d'organisa- } \\
\text { tion des acteurs locaux } \\
\text { - Manque d'image et de } \\
\text { reconnaissance du projet }\end{array}$ & $\begin{array}{l}\text { - Difficultés financières } \\
\text { liées à la complexité de } \\
\text { la procédure Interreg } \\
\text { - Difficultés politiques } \\
\text { entre les trois communes } \\
\text { - Difficultés pratiques } \\
\text { liées à la faiblesse des } \\
\text { infrastructures d'accueil }\end{array}$ \\
\hline
\end{tabular}

Tableau 3 : Les difficultés des projets culturels (Source : Hochedez C., 2004, enquêtes) Culturals projects and short comings

La population n'est jamais à l'origine d'un projet culturel, soit elle endosse l'habit du spectateur, de la partie prenante ou de l'opposant.

- Certains projets donnent à la population un rôle indispensable, tel le Festivache. Sans sa participation à la réalisation et à l'exposition des vaches en plein air, le projet n'existerait pas. La mise en place du concours de la plus belle vache constitue un facteur d'émulation. Le Festivache représente une réussite indéniable pour la participation de la population, au point que les organisateurs s'interrogent sur la conciliation entre participation de masse et production artistique. Le projet culturel oscille entre fête populaire (photo. 2) et manifestation artistique de plein air (photo. 3).

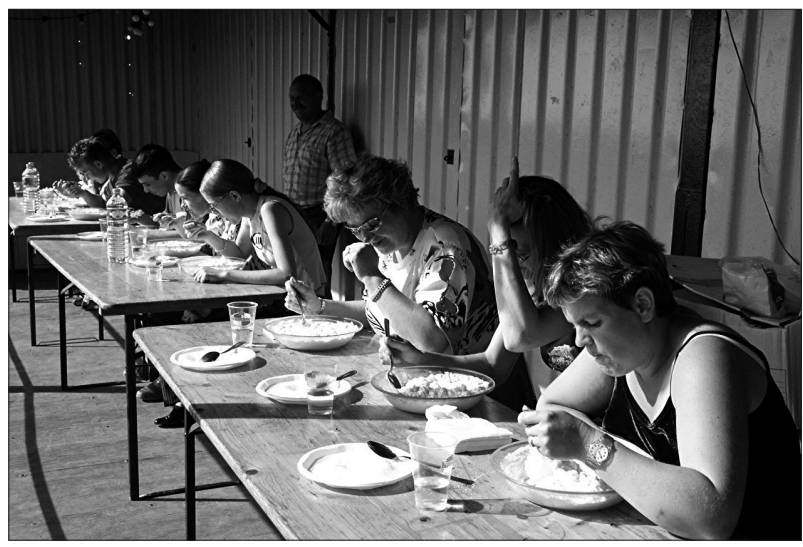

Photo 2 : Le concours du plus gros mangeur de fromage blanc (cl. M. Charlot, juillet 2003)

Cette animation est organisée dans le cadre de la journée du lait à Sains-Richaumont.

A local competion : who eats the largest amount of soft cheese?

This event takes place within the "day of milk" in Sains-Richaumont.

- Le milieu scolaire peut jouer un rôle d'effet levier et de diffusion important. Ainsi, l'Écomusée du textile et de la vie sociale de Fourmies trouve son origine dans un projet pédagogique mené par les écoles primaires du canton. En outre, les scolaires assurent une part importante de la fréquentation.

- Dans le cas des Transfrontalières, le rôle de la population est plus ambigu. L'un des points d'orgue de la saison a lieu le dimanche de Pentecôte lors du traditionnel défilé des chars réalisés par les associations locales. Cette animation sous-tend l'organisation d'un atelier d'insertion annuel, porté par le Centre Social d'Animation Culturelle de la ville d'Hirson. Mais les femmes et les hommes qui œuvrent à la réalisation des costumes et des chars ne sont conviés qu'aux manifestations populaires (défilé et concert gratuit du dimanche). La fréquentation régulière de la saison culturelle souligne, quant à elle, les fractures de la société thiérachienne, puisqu'elle est le fait des notables locaux, cadres et professions intellectuelles. Deux systèmes fonctionnent 
en parallèle sans se rencontrer, si ce n'est sous le terme générique de la manifestation. Ils caricaturent l'opposition entre culture populaire et culture élitiste. Qu'il s'agisse des Transfrontalières ou du Festivache, la fête semble constituer un vecteur propice à la participation de la population.

- Le projet Utopia entraîne l'opposition farouche de la population locale. Les raisons du blocage semblent assez évidentes. On pourrait arguer que l'opposition se porte sur le refus d'un projet imposé d'en haut, par le Conseil Général. La raison tient plus à l'enjeu social constitutif du projet. En effet, le projet de rendre à ce haut-lieu de l'utopie sociale sa dimension symbolique défend la patrimonialisation du site (photo. 4). Les habitants, retraités de l'usine Godin et veuves d'anciens ouvriers, souvent âgés et de condition modeste, refusent farouchement de quitter leurs logements. Pour eux, le Familistère est un patrimoine immobilier et affectif, alors que pour les concepteurs du projet il représente un patrimoine architectural et un témoin scientifique. Les deux approches s'opposent à tel point qu'on ne parle plus jamais du projet mais de la polémique qu'il suscite avec les habitants du familistère, constitués en association « Pour le droit de continuer à vivre au Palais social ».

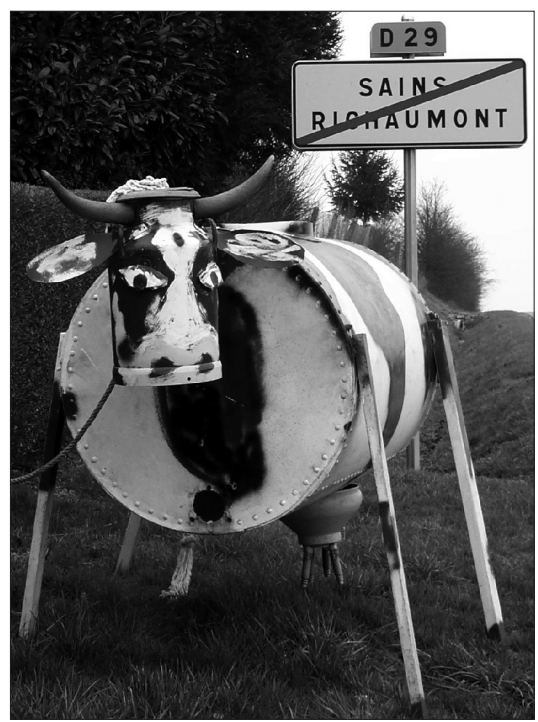

Photo 3 : Vache artistique en plein air (cl. C. Hochedez, février 2004)

Cette vache réalisée avec des bidons métalliques reste installée à l'entrée du village neuf mois après la fin du Festivache.

Open Air Work of Art Cow

This cow, made out of old metal barrels will stay at the entrance of the village nine monthe after the "festivache" event is closed.

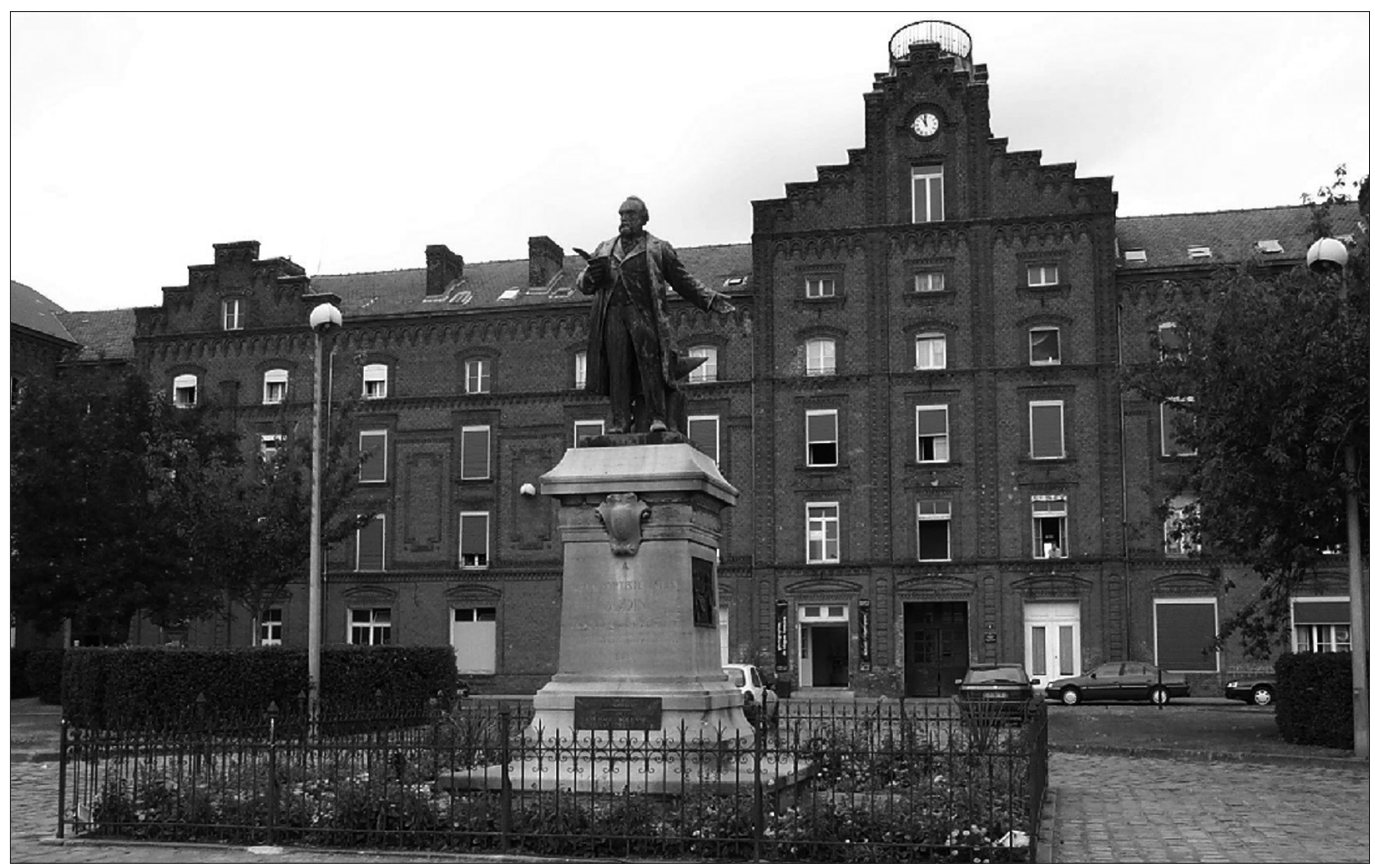

Photo 4 : Le pavillon central du Familistère Godin à Guise (cl. C. Hochedez, juillet 2003) Centerbuilding of the Godin Familistère in Guise 


\section{Conclusion : La quête impossible du géosymbole}

La réussite du projet culturel en termes de construction de lien social tient également, et peut-être avant tout, au géosymbole mobilisé, au sens défini par Joël Bonnemaison (2000) de « la structure symbolique d'un milieu, d'un espace ». À ce titre, le projet culturel qui apparaît comme le plus abouti est celui du Festivache. Les porteurs du projet reconnaissent la facilité et la rapidité à fédérer les populations autour du thème de la vache. Comme si «l'animal aux yeux si doux » (Micoud, 2003) réifiait un des mythes fondateurs du territoire, associé à celui d'un âge d'or de la Thiérache, d'une prospérité économique fondée sur l'élevage. Même si le projet culturel de l'Écomusée est aujourd'hui entré dans sa phase de maturité, au point que l'on puisse se demander s'il s'agit toujours d'un projet, les débuts n'ont pas été des plus faciles. Seule l'intervention du maire de Fourmies a réussi à dépasser les oppositions entre la sphère patronale encline à la mise en place d'un seul musée des techniques, les syndicats opposés à la muséification des luttes ouvrières et des rudes conditions de travail des ouvriers textiles et une population locale indifférente.

Si l'on considère les projets culturels menés plus généralement en Thiérache, on note que les projets valorisant le patrimoine que l'on qualifiera de rural, agricole et naturel, rencontrent plus de facilité et de reconnaissance que les projets à dimension industrielle, reléguant dans l'oubli une part constitutive de la société et du territoire. Au final, on peut considérer que l'assise territoriale des quatre projets étudiés correspond également à un ancrage dans des territoires culturels différenciés, réactivant la coupure entre Thiérache agricole et rurale et Thiérache industrielle et urbaine.

\section{Bibliographie}

Amas (V.), 1997. - La culture est-elle un facteur de développement territorial pour l'Avesnois?, mémoire de maîtrise, UFR de géographie de Lille 1, 118 p.

BonERANDi (E.), 2004. - « La mobilité des populations pauvres dans les espaces anciennement industrialisés. Pour une relecture de la pauvreté en milieu rural ", actes du $2^{\mathrm{e}}$ colloque franco-espagnol de géographie rurale, Habiter et vivre dans les campagnes de faible densité, Foix, 15-16 septembre 2004, ClermontFerrand, Presses universitaires de Clermont-Ferrand, p. 703-722.

—, 1999. - « La Thiérache, un espace intermédiaire entre crise et mutation », Hommes et Terres du Nord, $\mathrm{n}^{\circ} 2$, p. 85-96.

Bonnemaison (J.), 2000. - La géographie culturelle, cours de l’Université Paris IV-Sorbonne, 1994-1997, Paris, CTHS, $152 \mathrm{p}$.

Crozat (D.), 1998. - Géographie du bal en France : diversité régionale. La production culturelle de l'espace local : acteurs, spécificités et identités culturelles, territoires ou réseaux, Thèse de géographie, Université Lyon 2 Louis Lumière, 400 p. + annexes 200 p.

Delfosse (C.), 2003. - Géographie rurale, culture et patrimoine, HDR, Université de Lille 1, 2 vol., 312 p. et $443 \mathrm{p}$.

Di Méo (G.), 2001. - La géographie en fêtes, Paris, Ophrys, 265 p.

Dubois (V.), 1995. - Le patrimoine au service du développement territorial transfrontalier, l'exemple de l'Avesnois-Thiérache: vers une eurorégion rurale, DESS, UFR Géographie et d'Aménagement de Lille 1, 85 p.

Hochedez (C.), 2004. - Des machines, des vaches et des hommes. Projets culturels, acteurs et territoires dans un espace rural en crise : l'exemple de laThiérache, mémoire de Maîtrise, UFR géographie, Lyon 2, 217 p. 
JEAN (Y.), 2001. - «Être rural à la fin du XXe siècle », dans Faire la géographie sociale aujourd'hui, Caen, Les documents de la MRSH de Caen, $n^{\circ}$ 14, p. 111-122.

Ministère de la Culture et de la Communication, 1998. - Atlas des activités culturelles, Paris, La Documentation Française, 210 p.

Micoud (A.), 2003. - « Ces bonnes vaches aux yeux si doux », Communications, n 74, p. 217-237.

Rigaud (J.), 1996. - Pour une refondation de la politique culturelle, Rapport au ministère de la Culture, Paris, La Documentation Française, 201 p.

Moulinier (P.), 2003. - Politique culturelle et décentralisation, Paris, L'Harmattan, 336 p.

SAEZ (J.-P.) (dir.), 1995. - Identités, cultures et territoires, Paris, Desclée de Brouwer, 267 p.

Virassamy (C.), 2002. - Les pôles d'économie du patrimoine, Paris, DATAR/La Documentation Française, $90 \mathrm{p}$.

Cet article a été reçu le 20 novembre 2006 et définitivement accepté le 21 mai 2007. 
